\title{
FONDATION DE QARTHADASHT EN AFRIQUE DU NORD - COMME PROBLEME DANS LES RECHERCHES SUR LA CHRONOLOGIE DE LA PLUS ANCIENNE HISTOIRE DE CARTHAGE
}

\author{
MiRON WOLNY \\ University of Warmia and Mazury in Olsztyn (Poland) \\ miron.w@wp.pl
}

\begin{abstract}
The author of the article tries to connect the observation of economic and trade relations developed by the Phoenicians in the western part of the Mediterranean with a reflection on the situation in which the Levant countries found themselves. It is known that in the period in which the founding of Carthage can be hypothetically located, the Phoenician centers were under political, economic and military pressure - mainly from Assyria - although other powers, such as Damascus, cannot be ruled out. On the other hand, however, it is known that, for example, in German science the lack of a founding act of Carthage in North Africa was emphasized, and the archaeological traces left in this territory seem insufficient to reconcile conventional literary relations with the founding of Carthage at the end of the 9 th century BC. The intention of this article is an attempt to show the issues on the basis of which one should consider the reinterpretation of the events reported as the context of the founding of Carthage. This procedure would serve to revise the existing findings of science on the chronology of the founding of Qarthadasht and could, consequently, contribute to showing that the founding of Carthage fell on a later period i.e. the end of the 8 th or the beginning of the 7 th century BCE.

KEYWORDS: History of Tyre, Phoenician Colonisation, Carthage, Ancient Historiography, Chronology of the founding of Qarthadasht.
\end{abstract}

L'importance de la colonie phénicienne de Carthage sur les territoires d'Afrique du Nord ne nécessite pas d'être soulignée de façon particulière - la contribution de cet élément culturel à l'image globale de la civilisation de la partie occidentale du bassin méditerranéen est fructueuse et clairement visible. ${ }^{1}$ L'affrontement civilisationnel avec Rome, qui est entré dans l'histoire sous le nom de Guerres

\footnotetext{
${ }^{1}$ Gsell 1920, 374-401; Ehrenberg 1965, 549-586; Fantar 1970; Acquaro 1978; Huss 1985; Mazza 1988, 548-568; Lancel 1992; Ameling 1993. 
Puniques, a conduit à la formation d'un certain nombre de convictions, qui, malheureusement, sont survenues principalement du côté du participant victorieux au conflit, ${ }^{2}$ perpétuant l'image standard de Carthage en tant que mastodonte sanguinaire dont l'apprivoisement est devenu une nécessité historique. ${ }^{3}$ Cela était corrélé à la thèse sur Carthage en tant qu'État au moins aussi ancien que Rome ou même plus ancien. ${ }^{4}$ Cette thèse, en termes généraux, est maintenue à ce jour, ce qui, avec toute l'attention portée aux recherches scientifiques, ne permet pas d'aller au-delà de la convention théorique concernant un tel constat. En plus, comme le résume avec précision W. Huss, déclarant que, d'une part, il est substantiellement justifié de s'appuyer sur les données provenant de Timée ou de Ménandre d'Éphèse, et d'autre part, les historiens ne possèdent pas de description crédible se référant à l'acte fondateur de Carthage. ${ }^{5}$ Face à une pénurie de découvertes d'une importance historique, cette situation créera toujours une sorte de liberté dans l'étude de la chronologie de la plus ancienne histoire de Carthage. L'intention de cet article est une tentative de déterminer les questions sur la base desquelles on devrait considérer la réinterprétation des événements rapportés comme le contexte de la fondation de Carthage. Cette démarche servirait à réviser les conclusions scientifiques actuelles sur la chronologie de la fondation de Qarthadasht.

La tradition de la navigation forcée par les circonstances de l'existence sur le littoral du Liban s'inscrivait dans la représentation complexe des Phéniciens, conservée à la fois par la tradition indigène et par les sources les plus anciennes associées à la tradition de l'antiquité grecque. ${ }^{6}$ C'est un point important qui permet, dans une certaine mesure, de recréer les grands traits du fonctionnement des Phéniciens dans la structure des voies commerciales, qui ont ensuite été utilisées par les Grecs. ${ }^{7}$ Les habitants et les représentants des centres phéniciens en développement progressif, ${ }^{8}$ en commençant par le plus ancien Byblos, en passant par Sidon, Tyr, Akko et Arados, s'ouvraient à la découverte de la partie occidentale du bassin de la Méditerranée, nouant lentement des liens avec eux en établissant des contacts commerciaux. On suppose qu'à partir du XIIe siècle av. J.-C., les relations avec les communautés d'outre-mer commencèrent à se développer de façon de plus en plus intense, ce qui a abouti à l'établissement de relations entre le

\footnotetext{
${ }^{2}$ Brizzi 2011.

${ }^{3}$ Kiernan 2004, 27-39; Miles 2010.

${ }^{4}$ Cornell 1997, 92-97.

${ }^{5}$ Huss 1985, 42-43.

${ }^{6}$ Latacz 1990,11-21.

${ }^{7}$ Bunnesi979.

${ }^{8}$ Delcor 1995, 333-346; Katzenstein 1973.
} 
Levant et le royaume de Tarshish (ou Tartessos) situé en Andalousie. ${ }^{9}$ Comme le souligne J. B. Tsirkin, entre le VIIIe et le VIe siècle av. J.-C., lorsque la plupart des colonies phéniciennes sont apparues dans le sud de l'Espagne, Tartessos était une puissance dans cette région. Les Phéniciens avaient des contacts assez étroits avec les habitants de cet empire, ce qui était impliqué par le fait que, en raison du pragmatisme existentiel, les deux parties avaient besoin l'une de l'autre. Comme le souligne Tsirkin, les habitants de Tartessos transmettaient aux Phéniciens des produits, qu'ils exportaient ensuite vers l'Orient, et les habitants de Tartessos, grâce à la mobilité des Phéniciens, pouvaient avoir accès aux marchés inépuisables de l'Orient, ce qui garantissait à l'aristocratie de Tartessos des bénéfices incommensurables. Castillo de Doña Blanca, situé en face de Gades était l'exemple d'une telle coexistence. ${ }^{10}$ La chronologie de la formation de cette colonie est problématique, même si son apparition dès le IXe siècle av. J.-C. ne peut être exclue." Ces relations ont probablement été l'un des facteurs les plus importants conduisant à l'initiation du mouvement phénicien vers l'Ouest, bien que l'intensification de cette activité ait bien sûr été un processus multifactoriel. L'une des conséquences les plus importantes du mouvement vers l'Ouest des Phéniciens fut la fondation de Carthage. ${ }^{12}$

La fondation par les Phéniciens de Tyr d'une nouvelle colonie en Afrique du Nord ne soulève pas de controverses majeures, même si dans le processus de fondation de Carthage, il convient également de prêter attention au rôle de Chypre. ${ }^{13}$ Le problème de la fondation de Carthage a été examiné à maintes reprises dans les recherches scientifiques, et une tentative de proposer une chronologie plus détaillée de l'acte fondateur était un objectif important de ces actions. Ici, le champ des alternatives semble relativement large, car théoriquement elles peuvent être situées entre le XIIIe et le VIIIe siècle av. J.-C., voire le VIIe siècle av. J.-C. En pratique, comme le souligne J. B. Tsirkin, Carthage a du être fondée trois cents ans après la fondation de Gades, ${ }^{14}$ dont nous ne pouvons pas situer les origines chronologiquement, remontant bien au-delà du premier millénaire av. J.-C.

Ainsi, le problème fondamental pour déterminer la chronologie de la fondation de Carthage réside dans la recherche de cohérence entre les matériaux archéologiques et l'ancienne tradition littéraire. Dans les deux cas, il s'agit d'une

\footnotetext{
${ }^{9}$ Schulten 1950, 42-44; Blázquez 1975, 272-283; Aubet 1982, 321-324.

${ }^{10}$ Tsirkin 1997, 243-244.

${ }^{11}$ Moscati 1989, 13-15; Tsirkin 1997, 243.

${ }^{12}$ Carayon 2008, 128-129.

${ }^{13}$ Baurain 1988, 15 ff.; Lemaire 2010, 55 .

${ }^{14}$ Tsirkin 1997, 249: Gades was built almost three hundred years earlier than Carthage.
} 
image incomplète de la réalité, ce qui implique l'élargissement de la sphère théorique du discours scientifique autour de la question de la chronologie de la fondation de Carthage. ${ }^{15}$ Cela indique que la longue tradition de cette colonie phénicienne, issue de sources littéraires, ne bénéficie pas d'un support adéquat dans le matériel archéologique. ${ }^{16}$ Certes, cette situation ne disqualifie pas complètement le message historiographique, mais elle provoque une sorte de gêne liée aux archives littéraires traditionnelles sur la création de Carthage.

Selon le message de Trogue-Pompée, répété dans l'œuvre de Justinus, le légendaire Dido (Élissa) - la fondatrice de Carthage, devait être la fille du roi tyrien Pygmalion (Pygmalione et Elissa filia). ${ }^{17}$ L'utilisation de cet indice a permis de situer en 824 av. J.-C. les événements liés à l'arrivée de Dido en Afrique du Nord. L'indice recommandé est également quelque peu cohérent avec les informations contenues chez Flavius Josèphe, qui parle la septième année du règne de Pygmalion, ${ }^{18}$ ce qui donnerait l'année 819 av. J.-C. Ces données, synchronisées avec la chronologie du règne des rois de Tyr, ont conduit à privilégier dans la science la date de 814 av. J.-C., également basée sur les données de Timée. ${ }^{19}$ Cependant, cela ne change rien au fait que la discussion sur ce problème peut difficilement être considérée comme achevée, car l'adoption d'une solution basée sur des synchronisations "recherchées", qui ne reposaient que sur des prémisses basées sur une trame légendaire, ne semble que partiellement satisfaisante. D'un autre côté, il est compréhensible que l'historien de l'Antiquité soit dans de nombreux cas obligé de recourir à ce type de démarches, en particulier dans les situations où il y a pénurie de preuves autres que les sources littéraires. Cette situation se complique lorsque les sources littéraires à la disposition du chercheur ne sont que la représentation d'une tradition qui surgit en dehors de la sphère de l'historiographie. Les situations de ce genre laissent nécessairement derrière elles les résultats obtenus dans le domaine théorique, leur permettant parfois de rester longtemps et à l'aise dans les connaissances académiques.

Cela ne change pourtant rien au fait que, dans le cas de Carthage, il s'agit d'un moment historique dans lequel devait commencer le processus de consolidation de l'élément phénicien, important sur le plan de la formation de culture en Afrique du Nord, avec des conséquences importantes, non seulement pour l'histoire ancienne, mais aussi pour l'histoire universelle. Face à ce fait, ainsi qu'à

\footnotetext{
${ }^{15}$ Cintas 1970.

${ }^{16}$ Guzzo Amadasi 1967, 1-11.

${ }^{17}$ Iust. 18.4.1.

${ }^{18}$ Flav. Contra Appionem 1.17.107-108; 18.117-126; por. Antiq. 8.3.1.

${ }^{19}$ Plb. 12.28a.3.
} 
la possibilité d'autres propositions, il est difficile de laisser dans la catégorie d'axiome l'affirmation que Carthage a été fondée au IXe siècle av. J.-C. ${ }^{20}$

Un regard global sur le problème de la colonisation phénicienne qui a abouti à la création de Carthage laisse l'offre de recherche limitée par des propositions extrêmes: la plus ancienne, datant du XIIIe siècle av. J.-C. qui ne peut être défendue par la méthodologie scientifique traditionnelle et la plus jeune, également appelée hypercritique, située au VIIe siècle av.J.-C., ou plus précisément dans les années $673-663$ av. J.-C. que la science moderne a cependant rejeté comme trop tardive. Les conclusions de F. Rakob ont probablement également été influencées par l'acquisition de matériel archéologique jusqu'alors inconnu. ${ }^{21}$ Cette situation, comme le souligne A. Lemaire, a eu un impact majeur sur la réduction des théories hypercritiques, qui pendant un certain temps pouvaient se baser sur la situation d'un déficit d'artefacts archéologiques (arguant éventuellement de l'absence de restes archéologiques archaïques). ${ }^{22}$ Le problème, auquel les scientifiques n'ont pas été en mesure de répondre jusqu'à présent, c'est avant tout à quel moment l'action de précolonisation a pris fin et, par conséquent, quand a eu lieu la fondation proprement dite de Carthage en tant que comptoir commercial indépendant. Après tout, la précolonisation est un phénomène bien connu. Strabon informe, par exemple, sur la reconnaissance du terrain par les Tyriens ( $\tau 0 ن ่ \varsigma ~ \delta \dot{~} \pi \varepsilon \mu \varphi \theta \dot{v} v \tau \alpha \varsigma$

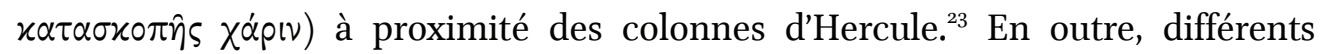
témoignages littéraires sur les activités de précolonisation sont fournis, par exemple, par l'Odyssée, où dans l'un des extraits ${ }^{24}$ il y a une mention sur un navire du peuple phénicien habile dans la navigation quoique bien avide transportant diverses marchandises à des fins commerciales:

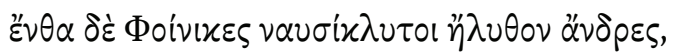

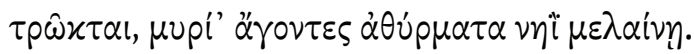

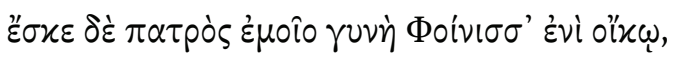

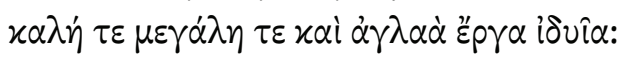

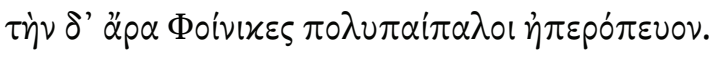

Il ressort clairement de l'extrait cité qu'il s'agit ici d'une expédition dont le but était la vente immédiate de marchandises et en aucun cas cette action ne peut

\footnotetext{
${ }^{20}$ Lancel 1992, 32-48.

${ }^{21}$ Rakob 1989, 155-208.

${ }^{22}$ Lemaire 2010, 56 .

${ }^{23}$ Strab. $3 \cdot 5 \cdot 5$.

${ }^{24}$ Od. 15.418-420.
} 
être combinée avec des engagements visant à s'installer sur un territoire donné. Cette situation semble également confirmée par un autre fragment de l'Odyssée, ${ }^{25}$ qui évoque le séjour annuel des Phéniciens sur le territoire d'où s'exerçait l'activité commerciale - consistant à acheter de nombreuses marchandises:

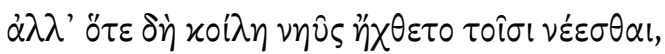

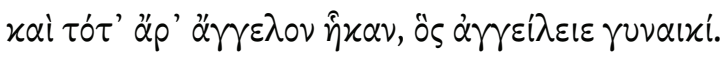

Si ces indications renvoient à un certain mécanisme de fonctionnement commun, dont l'essence était l'activité marchande, alors en dehors des mentions dans les plus anciens rapports littéraires il restaient des traces matérielles de cette activité de précolonisation. Il ne peut être exclu qu'avant la fondation des colonies phéniciennes - y compris Carthage, la pratique du peuple marin ait pu être une tradition bien plus longue de reconnaissance de terrain, comme le souligne W. Cullican, sur la base des temoignages de Diodore. Néanmoins, ce qui est important, Cullican admet à juste titre que nous ne connaissons pas vraiment les suppositions des auteurs antiques sur la position dominante des Phéniciens à l'Ouest (But we do not know what presuppositions ancient authors had about Phoenician precedence). ${ }^{26}$

Dans un passage plus long sur les origines de l'apparition des Phéniciens en Sicile, Thucydide semble démontrer la connaissance du processus plus long qui a conduit à leur prise de possession d'une partie de l'île. L'auteur grec, avec son exclusivité inhérente, conclut au résultat de ce processus, qui était l'arrivée de ces

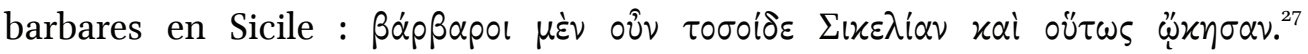
L'expression de cette pensée est cependant précédée par l'information selon laquelle les Phéniciens ont d'abord occupé les promontoires et les îles adjacentes à la Sicile afin de pouvoir faire du commerce avec les Siciliens. Cependant, comme de plus en plus de Grecs ont commencé à arriver par la mer, les Phéniciens ont quitté la plupart de leurs colonies et se sont retirés à Motie, Soloeis et Panormos. En conséquence, ils se sont retrouvés près des Elims, où ils se sentaient en sécurité car ils avaient de bonnes relations avec eux. D'ailleurs, comme en informe plus loin Thucydide, ils se sont retrouvés à une courte

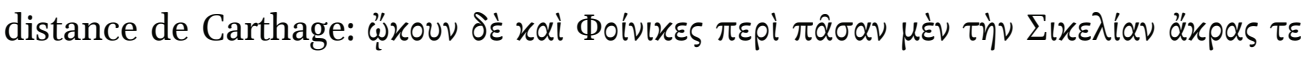

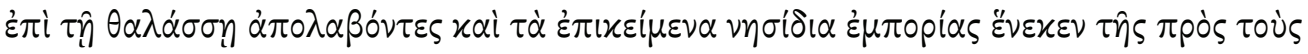

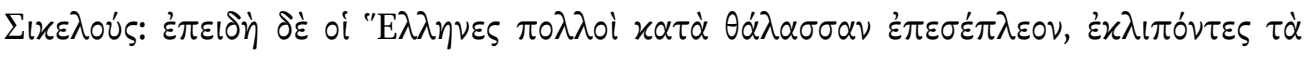

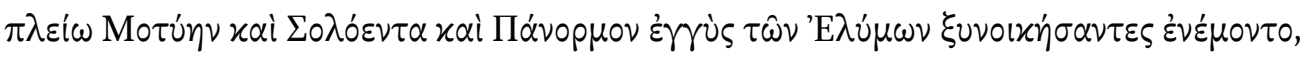

\footnotetext{
${ }^{25} \mathrm{Od} \cdot 15 \cdot 459-460$.

${ }^{26}$ Culican 1991, 486-492.

${ }^{27}$ Thuc. 6.2.6.
} 


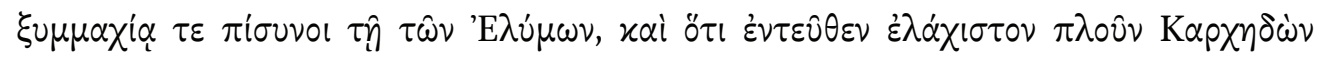

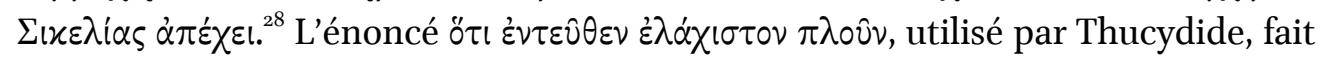
référence à une route maritime et indique la distance qui peut être parcourue. Dans le même temps, il semble découler du contexte situationnel global que lorsque les Phéniciens se sont installés en Sicile, Carthage existait déjà, ce qui serait également cohérent avec le fait que leur mouvement dans cette direction était sur le point de commencer au moment où le mouvement de colonisation grecque a commencé ou s'est intensifié. ${ }^{29}$ Cependant, il ne peut être exclu que Thucydide, en écrivant sur Carthage, voulait parler de l'endroit où l'État existait à son époque.

La situation ci-dessus semble influencer dans une certaine mesure une vue d'ensemble du matériel archéologique avec lequel les scientifiques auraient voulu prouver la fondation antérieure de Carthage. Par rapport à l'ensemble des agissements de colonisation des Phéniciens en Occident, W. Culican souligne que les conclusions tirées de l'analyse des découvertes d'artefacts ne sont pas particulièrement utiles ici. À titre d'exemple, il cite une statue en bronze de Baal, extraite de la mer au large des côtes de Sciacca, dans le sud-ouest de la Sicile, qui ne serait guère considérée comme une preuve solide de l'installation des Phéniciens en Occident. Cette trouvaille est comparée aux figurines cananéennes de l'âge de bronze, ce qui suggère qu'elle a peut-être parcouru les mêmes routes le long des voies commerciales mycéniennes, par lequelles les vases funéraires chypriotes de l'âge de bronze se sont retrouvés dans l'est de la Sicile (could well have come by the same, presumably Mycenaean, channels which brought Cypriot vases to Bronze Age graves in eastern Sicily). ${ }^{30}$ En indiquant d'autres exemples potentiels, Culican conclut enfin qu'il n'y a généralement aucune preuve pour reconstruire la totalité des contacts précoloniaux (the picture of pre-colonial contact, for which generally there is a remarkable lack of evidence)..$^{31}$ Cette exemplification est étayée par un certain nombre d'autres données archéologiques, ce qui laisse un champ assez large d'alternatives sur le plan de la chronologie concernant la fondation de colonies phéniciennes respectives. ${ }^{32}$ Pour cette raison, il serait difficile d'exclure Carthage du processus général de précolonisation et de colonisation proprement dite.

\footnotetext{
${ }^{28}$ Thuc. 6.2.6.

${ }^{29}$ Dunbabin 1948, 327-329.

${ }^{30}$ Culican 1991, 486: the picture of pre-colonial contact, for which generally there is a remarkable lack of evidence.

${ }^{31}$ Culican 1991, 486.

${ }^{32}$ Cintas 197 o.
} 
Il convient de souligner à cet endroit que l'établissement des paradigmes des recherches dans le domaine de l'analyse des artefacts et des rapports littéraires sur le mouvement migratoire des Phéniciens devrait également être influencé par la tendance avérée de ce peuple à souligner sa propre identité. Selon les informations d'Arrian, que nous retrouvons également chez Quinte-Curce, ${ }^{33}$ les habitants de Tyr ont décidé de refuser fermement de laisser Alexandre entrer dans la ville alors qu'il avait l'intention de faire des offrandes dans le temple de Melqart. J. B. Tsirkin souligne que ce refus était en grande partie dû à des considérations politiques, mais que des raisons religieuses ne devraient pas non plus être exclues. ${ }^{34}$ Cela montre que les Phéniciens avaient une préoccupation profondément enracinée pour leurs propres spécificités culturelles. Dans l'ensemble, cela semble également éclairer le processus bien prudent d'assimilation des Phéniciens dans la partie occidentale du bassin de la Méditerranée. Au cours de ce processus, un mécanisme a été développé pour distinguer des traits clairs de la culture phénicienne, qui en particulier dans son aspect punique, était soumise au processus relativement tardif d'hellénisation. ${ }^{35}$ De plus, cette culture a survécu et n'a pas non plus succombé facilement aux influences romaines, ce qui est également visible après les guerres puniques. ${ }^{36}$ Ainsi, les facteurs de perennité de la culture punique étaient des facteurs internes, pas nécessairement dépendants de la chronologie plus ou moins longue de l'existence de Carthage. En effet, adopter une perspective historique plus courte de Carthage ne limite en rien la fécondité de son influence, et qui plus est, semble travailler considérablement à son avantage.

Le maintien d'une perspective historique plus courte de Carthage semble résulter non seulement de la perception de la situation dans le bassin de la Méditerranée occidentale (y compris une réflexion sur le matériau d'artefact préservé reflétant la vision des relations commerciales en Méditerranée occidentale du IXe au VIIe siècle av. J.-C.), mais également de l'analyse de la pression exercée sur les Phéniciens par leurs pénibles voisins. Dans cet aspect, il serait difficile d'ignorer l'analyse hypercritique qui subordonne l'interprétation

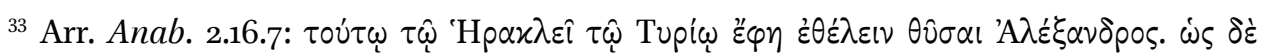

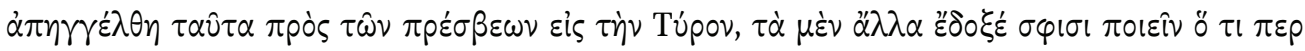

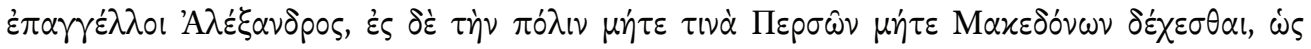

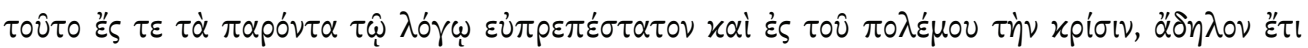

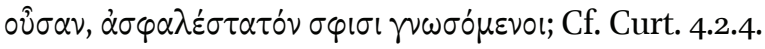

${ }^{34}$ Tsirkin 1997, 244: Of course, the refusal was largely prompted by political reasons, but religious considerations should not be excluded either.

${ }^{35}$ Luria 1964, 53-75.

${ }^{36}$ Campus 2012, 24-32, 97-111.
} 
des données littéraires à l'étendue représentative du matériel archéologique, et de plus, renvoie également à l'analyse des motifs de dynamisation du mouvement migratoire qui a eu lieu dans les centres phéniciens d'origine. Dans son mémoire de synthèse, Frézouls a récapitulé la théorie qui, grâce à la réinterprétation des traditions littéraires, avec l'implication de sources assyriennes, déplace la fondation de Carthage au VIIe siècle av. J.-C. ${ }^{37}$ Ce postulat de recherche était basé sur la théorie avancée par E. O. Forrer, supposant un champ d'alternatives à la fondation de Carthage entre 673 et 663 av. J.-C. ${ }^{38}$ Le savant a fondé ses réflexions sur l'analyse des traditions des sources assyriennes, indiquant deux événements importants de l'histoire de Tyr, sous les règnes d'Assarhadon et d' Ashurbanipal. Le premier était lié au siège de Tyr par Assarhadon (671/670 av. J.-C.), à la suite duquel, une partie de la famille royale est tombée en captivité assyrienne. Après le deuxième blocus de Tyr, rendu effectif après 666 av. J.-C., le souverain tyrien, Ba'al, a été livré à Ashurbanipal. Forrer pense donc que les deux filles de Ba'al, Anna et Didon, ont échappé aux Assyriens et ont fui vers l'Ouest. Il faut admettre que cette histoire, d'ailleurs, correspond bien à l'image de l'expédition, qui, pour échapper aux Assyriens, devient une expédition vers un lieu lointain et peut-être déjà connu (c'est là que revient le problème de la précolonisation soulevé auparavant). Forrer, de façon orthodoxe, défend ces événements comme liés à la première fondation de Carthage. Il indique que le placement de la fondation de Carthage sous le règne de Pygmalion est erroné, car dans ce cas il s'agit probablement du Qarthadasht chypriote, ${ }^{39}$ c'est-à-dire le Kition conquis par les Tyriens en 814 av. J.-C. Ce qui donne encore plus de piquant à cette histoire c'est le fait que celle dont Virgil Didon se souvient est la fille de Belus (Ba'al) et non de Pygmalion. Cela ne parait pas déraisonable puisque nous trouvons la même ascendance également chez Silius Italicus. ${ }^{40}$ Bien que nous utilisions à nouveau un argument littéraire, il est difficile de trouver ici des contre-arguments totalement convaincants.

L'essentiel de la question, cependant, ce sont les questions problématiques du déficit de preuves archéologiques, ainsi qu'une tentative de considérer les circonstances de la fondation de Carthage dans la perspective d'un contexte historique plus large. Alors que le matériel archéologique obtenu en Afrique du Nord a une certaine perspective de développement, qui, après tout, ne doit pas être suffisante pour soutenir la fondation de Carthage au IXe siècle av. J.-C. sans aucun doute, l'indication des facteurs internes qui ont conduit les Phéniciens à

\footnotetext{
${ }^{37}$ Frézouls 1955, 153-176.

${ }^{38}$ Forrer 1953, 85-93.

${ }^{39}$ Lipiński 1966, 209-234.

${ }^{40}$ Sil. 1.8 o ff.
} 
quitter leur colonie Tyrienne, pourrait éclairer davantage les conditions de colonisation conduisant à l'essor de Carthage. La proposition formulée par A. Lemaire vise à examiner la pression des Assyriens sur le Levant, et son objectif est de répondre à la question du rôle réel de la politique des souverains assyriens dans l'intensification des mouvements migratoires phéniciens. ${ }^{41}$ Le savant français a observé que la pression politique et économique de l'empire néoassyrien sur les centres phéniciens ne s'est manifestée principalement que sous le règne de Tiglat-Phalazar III, c'est-à-dire dans les années 744-727 av. J.-C. Les agissements précédents des Assyriens sur ces territoires étaient sous forme de pillage, ${ }^{42}$ et les actions confirmées de Salmanazar III (858-824 av. J.-C.) et d' Adadanirari III (810-783 av. J.-C.) ont conduit à recueillir l'hommage de guerre, mais n'ont pas déterminé les centres phéniciens comme tributaires réguliers. ${ }^{43}$ Pour cette raison, comme le prétend A. Lemaire, il semble peu probable que la fondation de Carthage puisse être interprétée comme la réaction de Tyr à la pression néo-assyrienne. ${ }^{44}$ En même temps, Lemaire précise que cela ne signifie pas qu'il n'y ait pas eu une telle pression, ${ }^{45}$ mais qu'il la voit ailleurs, en indiquant les actions du roi de Damas, Hazaël $\left(843-805\right.$ av. J.-C.), ${ }^{46}$ qui, on le sait, a réussi à conquérir rapidement tout le Levant.

Si nous devions rechercher les causes de l'activité de colonisation en lien avec la pression politique, alors les recherches effectuées par A. Lemaire ouvrent une nouvelle porte sur la direction des recherches de l'acte fondateur de Carthage dans les étapes chronologiques plus récentes, car la pression économique et politique de l'Assyrie ne fera qu'augmenter dans les années suivantes. Ce n'est

\footnotetext{
${ }^{41}$ Lemaire 2010, 57-59.

${ }^{42}$ Culican 1991, $467 \mathrm{ff}$. souligne la pression politique et militaire. Comme le souligne le scientifique, c'est l'augmentation, et non la baisse du niveau de vie dans leurs villes d'origine, qui pourrait inciter les premiers colons à répondre aux besoins du marché des métaux et autres matières premières. L'auteur estime également que l'intérêt croissant des Assyriens pour la Phénicie aurait pu stimuler ce marché. Dans le même temps, il indique qu'à part Chypre, la Phénicie n'avait aucun intérêt colonial significatif avant 700 avant notre ère.

${ }^{43}$ Röllig 1982, 20-28.

${ }^{44}$ Lemaire 2010, 58: Dans ces conditions, il paraît assez invraisemblable que la fondation de Carthage puisse être interprétée comme la réaction de Tyr à la pression néo-assyrienne puisque cette fondation a eu précisément lieu au moment où cette pression néo-assyrienne, jusqu'ici très faible, a pratiquement disparu.

${ }^{45}$ Comme une sorte de truisme, on peut répéter l'argument bien connu sur la puissance militaire de l'Assyrie - et l'excellente organisation de l'État à cet égard: Backer 2014, 19-47.

${ }^{46}$ Lemaire 1991, 91-108; Lemaire 2010, 58.
} 
peut-être pas une tentative inutile de valoriser des théories plus anciennes et d'essayer de les réconcilier avec les découvertes faites dans le domaine archéologique. On ne peut pas exclure l'idée que la pression de Hazaël ait déclenché le mouvement «d'évacuation » des Phéniciens, les conduisant à travers Chypre jusqu'aux côtes de l'Afrique du Nord, et que les dernières années aient permis de vérifier la fonctionnalité de cette route afin de terminer ce processus par l'acte de fondation de Carthage, alors qu'il y avait effectivement une pression lancinante de la part de l'Assyrie.

\section{BIBLIOGRAPHIE}

Acquaro, E. (1978) Cartagine. Un impero sul Mediterraneo. Roma.

Ameling, W. (1993) Karthago. Studien zu Militär, Staat und Gesellschaft. München.

Aubet, M. E. (1982) „Zur Problematik der orientalisierenden Horizontes auf der Iberischen Halbinsel", in: H. G. Niemeyer, ed. Phönizier im Westen. Mainz am Rhein, 321-324.

Baurain, C. (1988) „Le rôle de Chypre dans la fondation de Carthage”, in: E. Lipiński, ed. Carthago, (Studia Phoenicia 6). Leuven, 15-27.

Blázquez, J. M. (1975) Tartessos. Salamanca.

Brizzi, G. (2011) Metus Punicus. Studi e ricerche su Annibale e Roma. Bolonia.

Bunnes, C. (1979) L'expansion phénicienne en Méditerranée. Bruxelles/Rome.

Campus, A. (2012) Punico - Postpunico: Per una archeologia dopo Cartagine. Tivoli.

Carayon, N. (2008) Les ports phéniciens et puniques. Géomorphologie et infrastructures. Strasbourg.

Cintas, P. (1970) Manuel d'archéologie punique I, Histoire et archéologie comparées. Chronologie des temps archaïques de Carthage et des villes phéniciennes de l'Ouest. Paris.

Cornell, T. J. (1997) The Beginnings of Rome. Italy and Rome from the Bronze Age to Punic Wars (c. 1000-264 BC). London.

Culican, W. (1991) „Phoenicia and Phoenician Colonisation”, in. J. Boardman, I. E. S. Edwards, N. G. L. Hammond, eds. The Assyrian and Babylonian Empires and other States of the Near East, from the Eighth to the Sixth Centuries B.C. (The Cambridge Ancient History II, 3.2). Cambridge, 461-546.

De Backer, F. (2014) „The Smallest Neo-Assyrian Combat Unit”, Historiae 11, 19-47.

Delcor, M. (1995) „La fondation de Tyr selon l'histoire, l'archéologie et la mythologie. Le problème de l'identité d'Usu", in: M. H. Fantar, M. Ghaki, eds. Actes du IIIe Congrès International des Études Phéniciennes et Puniques. Tunis, 333-346.

Dunbabin, T. J. (1948) The Western Greeks. Oxford.

Ehrenberg, V. (1965) Karthago. Ein Versuch weltgeschichtlicher Einordnung, w: Polis und Imperium. Zürich, 549-586.

Fantar, M. (1970) Carthage. La prestigieuse cite d'Élissa. Maison Tunisienne. 
Forrer, E. O. (1953) Karthago wurde erst 673-663 v. Chr. begründet, in: H. Kusch, ed. Festschrift F. Dornseiff. Leipzig, 85-93.

Frézouls, E. (1955) „Une nouvelle hypothèse sur la fondation de Carthage”, $B C H$ 79, 153176.

Gsell S. (1920) Histoire ancienne de l'Afrique du Nord I: Les conditions du développement historique, les temps primitifs, la colonisation phénicienne et l'empire de Carthage. Paris.

Guzzo Amadasi, M. G. (1967), Le iscrizioni fenicie e puniche delle colonie in Occidente. Roma.

Huss, W. (1985) Geschichte der Karthager. München.

Katzenstein, H. J. (1973) The History of Tyre. Jérusalem.

Kiernan, B. (2004) „The First Genocide: Carthage 146 BC”, Diogenes 203, 27-39.

Lancel, S. (1992) Carthage. Fayard.

Latacz, J. (1990) „Die Phönizier bei Homer”, in: U. Gehring, ed. Die Phönizier im Zeitalter Homers, Mainz, 11-21.

Lemaire, A. (1991) „Hazaël de Damas, roi d'Aram”, in: D. Charpin, F. Joannès, eds. Marchands, diplomates et empereurs, études sur la civilisation mésopotamienne offertes à Paul Garelli. Paris, 91-108.

Lemaire, A. (2010), „Remarques sur le contexte historique et culturel de la fondation de Carthage", in: M. H. Fantar, ed. Carthage et les autochtones de son empire du temps de Zama. Tunis, 55-6o.

Lipiński, E. (1966) „La Carthage de Chypre”, Studia Phoenicia 1-2, 209-234.

Mazza F. (1988) „Wie die alte Welt die Phönizier sah”, in: S. Moscati, ed. Die Phönizier. Hamburg, 548- 568.

Miles, R. (2010) Carthage Must be Destroyed. The Rise and Fall of an Ancient Mediterranean Civilization. Allen Lane.

Rakob, F. (1989) „Karthago. Die frühe Siedlung: neue Forschungen”, Mitteilungen des Deutschen Archäologischen Institut Rom 96, 155-208.

Röllig, W. (1982) „Die Phönizier des Mutterlandes zur Zeit der Kolonisierung”, in: H. G. Niemeyer, ed. Phönizier im Westen. Mainz am Rhein, 20-28.

Schulten, A. (1950) Tartessos. Ein Beitrag zur ältesten Geschichte des Westens. Hamburg.

Tsirkin, J. B. (1997) „The Phoenicians and Tartessos”, Gerión 15, 243-251. 